\title{
Non traumatic rotatory subluxation of atlanto-axial joint in a child with severe pharyngitis and concomitant meningitis
}

\author{
K N H Thalagahage ${ }^{1}$, Manouri P Senanayake ${ }^{2}$, Hasitha Liyanaarachchi ${ }^{3}$, I N A Gooneratne ${ }^{4}$ \\ Sri Lanka Journal of Child Health, 2012; 41(2): 94-96
}

(Key words: Grisel syndrome; torticollis; meningitis; pharyngitis)

\section{Introduction}

Non-traumatic rotatory subluxation of atlanto-axial joint (or Grisel syndrome) is a rare complication of severe pharyngeal inflammation and is described predominantly in children ${ }^{1,2}$. We report a child with severe pharyngitis and meningitis who developed non-traumatic rotatory subluxation of atlanto-axial joint. Grisel syndrome with concomitant meningitis is extremely rare and can further delay diagnosis causing serious long term sequelae such as quadriplegia ${ }^{3}$.

\section{Case report}

A previously healthy five year old boy developed upper respiratory symptoms and pain on swallowing followed by severe neck pain and grossly restricted neck movements of two days duration. There was no history of trauma. He had taken oral amoxicillin for two days.

Physical examination found a febrile, ill-looking child with torticollis and exquisitely tender, enlarged upper cervical lymph nodes. Visualization of inflamed throat was difficult due to limited mouth opening. Tympanic membranes were not inflamed. He was treated for septic cervical lymphadenitis with intravenous penicillin and cloxacillin but two days later developed neck stiffness, headache, photophobia and positive Kernig sign without any other abnormal neurological signs.

${ }^{1}$ Intern House Officer, Professorial Unit, Lady
Ridgeway Hospital, Colombo, ${ }^{2}$ Professor in
Paediatrics, Faculty of Medicine, Colombo,
${ }^{3}$ Registrar, Professorial Unit, Lady Ridgeway
Hospital, Colombo, ${ }^{4}$ Consultant Radiologist, Lady
Ridgeway Hospital, Colombo

(Received on 20 May 2012: Accepted after revision on 15 June 2012)
Initial investigations found polymorphonuclear leucocytosis in peripheral blood (total white cells $25 \times 10^{9} / 1$; neutrophils $89 \%$ ) and elevated erythrocyte sedimentation rate $(132 \mathrm{~mm} / 1 \mathrm{st}$ hour $)$. Cerebrospinal fluid (CSF) contained polymorphs, lymphocytes and erythrocytes $(24,58$, and 37 per high power field respectively) with marginally raised protein $62 \mathrm{mg} / \mathrm{dl}$ (normal 20-40) and a sugar difference of $60 \%$. CSF culture and bacterial antigens for Streptococcus pneumoniae, Haemophilus influenzae and Neisseria meningitidis were negative.

Treatment with intravenous cefotaxime $(50 \mathrm{mg} /$ $\mathrm{kg} /$ dose) resulted in clinical cure but painful torticollis persisted. Upper and lower limbs were neurologically normal.

Inflammatory markers became normal. Cervical spine radiography showed subluxation of atlantoaxial joint; and on computerized tomography, anterior atlanto-dens interval was $5.9 \mathrm{~mm}$ (normal < $5 \mathrm{~mm}$ ) confirming atlanto-axial subluxation. Magnetic resonance imaging (MRI) showed thecal sac compression without spinal cord involvement. (Figures $1 \& 2)^{4}$.

Following diagnosis of Grisel syndrome and neurosurgical opinion he was managed conservatively with strict bed rest, cervical immobilization with a soft collar, analgesics and muscle relaxants (diazepam and baclofen). In two weeks neck movements became less painful but torticollis took over two months to resolve. Repeat imaging after complete clinical cure found anterior atlanto-dens interval to have returned to normal and thecal compression was absent. 


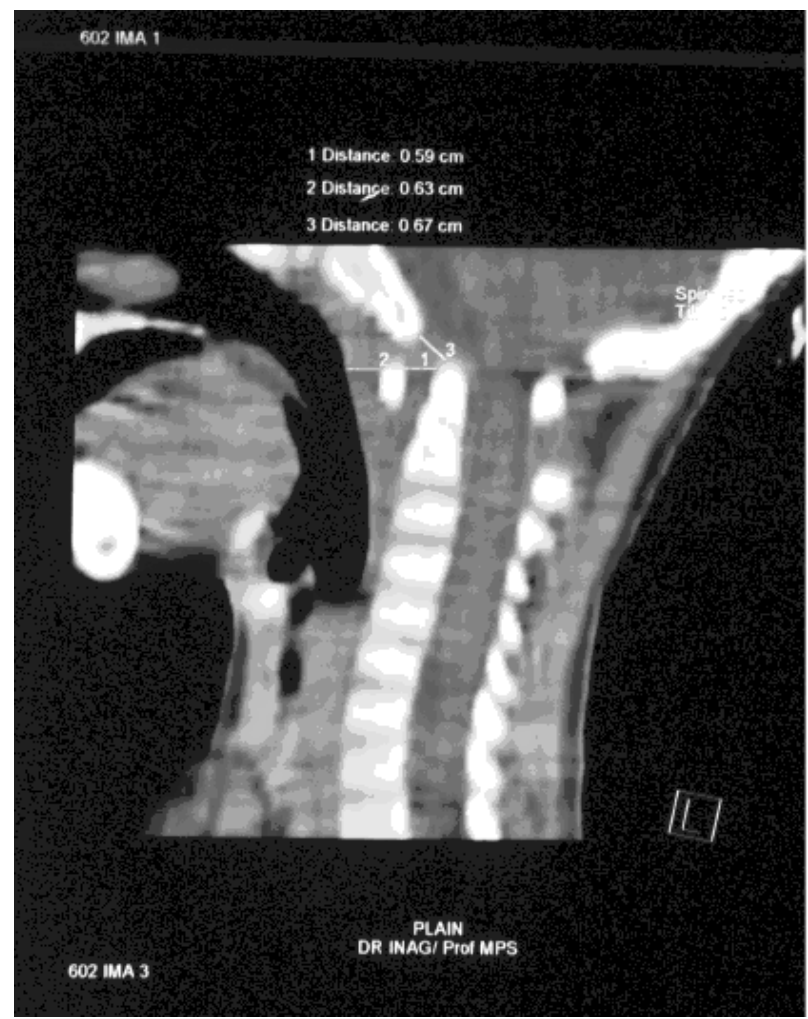

Figure 1: CT film showing increased $(5.9 \mathrm{~mm})$ atlanto-axial joint space marked No.1

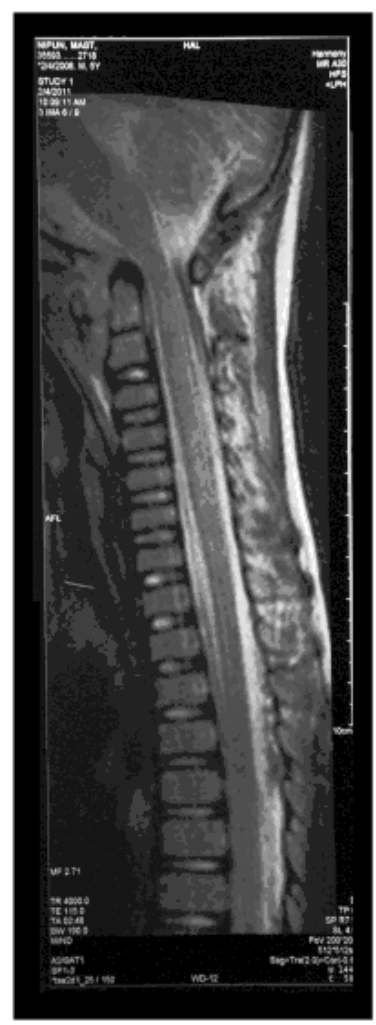

Figure 2: MRI showing thecal sac compression without spinal cord compression

\section{Discussion}

Grisel syndrome involves non-traumatic subluxation of the atlanto-axial joint due to inflammatory ligamentous laxity. This rare complication follows peri-pharyngeal infections or surgery and early diagnosis is of paramount importance to avoid long term neurological sequelae.

Our patient had upper respiratory tract infection which is the commonest infective cause reported ${ }^{1}$. Less common infective causes are retropharyngeal abscess and otitis media. Adeno-tonsillectomy is the commonest preceding surgical procedure ${ }^{1,2}$. A recent review has shown that post-infective cases are commoner than the better known, post -surgical causes ${ }^{1}$.

Lympho-venous anastomoses in the peri-pharyngeal area draining into the periodontal venous plexus is believed to be the anatomical basis for haematogenous transport of peri-pharyngeal sepsis to upper cervical structures and meninges. Laxity of the hyperaemic transverse ligament results in atlantoaxial subluxation. Spasm of the neck musculature due to severe pain may be a contributory factor.

This case highlights the need for a high degree of suspicion when a child presents with non traumatic torticollis in the presence of upper respiratory infections. Tenderness over second vertebra is suggestive and diagnosis is confirmed with CT and MRI imaging of neck.

In our patient clinical features of meningeal irritation were also present. Lack of microbial evidence on bacterial cultures and antigen tests was probably due to previous antibiotic therapy. We diagnosed pyogenic meningitis based on presence of polymorphs and significant sugar difference in CSF. Spread of head and neck space infections to meninges is a well-recognized sequel but Grisel syndrome associated with meningitis is extremely rare with only one previous report ${ }^{3}$. Early recognition and neuro-protective cervical spine stabilization have been shown to give a good outcome in Grisel syndrome $\mathrm{e}^{1,2,5}$.

This case highlights an important condition that paediatricians in particular should be mindful of because delay in diagnosis can result in quadriplegia or even death. 


\section{Acknowledgement}

We thank Dr. Jayantha Liyanage, Consultant Neurosurgeon, National Hospital of Sri Lanka for his assistance.

\section{References}

1. Karkos PD, Benton J, Leong SC, Mushi E, Sivaji $\mathrm{N}$, Assimakopoulos DA. Grisel syndrome in otolaryngology: A systematic review. International Journal of Pediatric Otorhinolaryngology 2007; 71, 1823-7. http://dx.doi.org/10.1016/j.ijporl.2007.07.002

2. Richter GT, Bower CM. Cervical complications following routine tonsillectomy and adenoidectomy. Current Opinions in Otolaryngology Head Neck Surgery 2006; 14 (6): $375-80$. http://dx.doi.org/10.1097/01.moo.0000247525.5 $\underline{6076.54}$

3. Wilson BC, Jarvis BL, Haydon RC. Nontraumatic subluxation of the atlanto-axial joint: Grisel syndrome Annals of Otology Rhinology \& Laryngology 1987; 96(6):705-8.

4. Wolfgang Dähnert Radiology Review Manual, 6th ed. Philadelphia, Pa: Lippincott Williams \& Wilkins, 2007: 195.

5. Samuel D, Thomas DM, Tierney PA, Patel KS. Atlanto-axial subluxation (Grisel syndrome) following otolaryngological diseases and procedures. Journal of Laryngology and Otology 1995; 109 (10): 1005-9.

http://dx.doi.org/10.1017/S0022215100131883 